\title{
Publisher Correction: Breastfeeding promotes bifidobacterial immunomodulatory metabolites
}

Christopher J. Stewart (D)

Correction to: Nature Microbiology https://doi.org/10.1038/s41564-021-00975-z, published online 21 October 2021.

In the version of this article initially published, there was an error in Fig. 1. Specifically, in the inset box "Aromatic amino acids," the word "Indolelactic" in the label "Indolelactic acid (ILA)" initially appeared as "Indoleacetic." The error has been corrected in the online version of the article.

Published online: 24 November 2021

https://doi.org/10.1038/s41564-021-01032-5

๑) Springer Nature Limited 2021 